\title{
Des Forges du Saint-Maurice aux fonderies de Montréal : mobilité géographique, solidarité communautaire et action syndicale des mouleurs, 1829-1881
}

\section{Peter Bischoff}

Volume 43, numéro 1, été 1989

URI : https://id.erudit.org/iderudit/304764ar

DOI : https://doi.org/10.7202/304764ar

Aller au sommaire du numéro

\section{Éditeur(s)}

Institut d'histoire de l'Amérique française

\section{ISSN}

0035-2357 (imprimé)

1492-1383 (numérique)

\section{Découvrir la revue}

\section{Citer cet article}

Bischoff, P. (1989). Des Forges du Saint-Maurice aux fonderies de Montréal : mobilité géographique, solidarité communautaire et action syndicale des mouleurs, 1829-1881. Revue d'histoire de l'Amérique française, 43(1), 3-29. https://doi.org/10.7202/304764ar

\section{Résumé de l'article}

À travers l'expérience des mouleurs originaires des Forges du Saint-Maurice, ce texte cherche à mieux cerner la participation des Canadiens français à la formation de la classe ouvrière, les relations entre mobilité géographique et activité syndicale, et le rôle du métier, de la famille et du voisinage dans les mouvements migratoires et l'adaptation des travailleurs à un nouveau milieu. L'étude des mouleurs dans le village des Forges du Saint-Maurice révèle l'existence de solidarités familiales et communautaires très intenses liant ces travailleurs depuis la fin du XVIII ${ }^{\mathrm{e}}$ siècle. Au cours des décennies 1830 et 1840, les mouleurs mettent en place les fondements d'un réseau communautaire qui s'étend à plusieurs localités du Québec, participant ainsi à l'expansion de l'industrie du moulage à travers la province.

À partir de la fin des années 1850, dans le contexte du déclin des forges mauriciennes et l'émergence de Montréal comme centre de la sidérurgie québécoise, leur réseau communautaire s'avère un outil essentiel dans leur migration et adaptation au milieu montréalais. Les mouleurs se concentrent graduellement dans la métropole, où ils prennent une part active à différentes actions revendicatives pour défendre ou promouvoir le statut de leur métier. Mais leur ardeur est longtemps tempérée par les tensions ethniques et linguistiques locales.
Tous droits réservés @ Institut d'histoire de l'Amérique française, 1989

Ce document est protégé par la loi sur le droit d'auteur. L'utilisation des services d'Érudit (y compris la reproduction) est assujettie à sa politique d'utilisation que vous pouvez consulter en ligne.

https://apropos.erudit.org/fr/usagers/politique-dutilisation/ 


\title{
DES FORGES DU SAINT-MAURICE AUX FONDERIES DE MONTRÉAL: MOBILITÉ GÉOGRAPHIQUE, SOLIDARITÉ COMMUNAUTAIRE ET ACTION SYNDICALE DES MOULEURS, 1829-1881 ${ }^{1}$
}

\author{
PETER BISCHOFF \\ Département d' histoire \\ Université de Montréal
}

\begin{abstract}
Forges St. Maurice
24 janvier 1846

... le premier octobre 1760 , des instructions du Général Amherst... furent communiquées... à un M. Courval aux dites Forges St Maurice, le priant de retenir... les ouvriers dont les noms étaient à la suite des dites instructions.

... les dits ouvriers étaient nommés Delorme, Robichon, Marchand, Humblot, Serreau, Tassé, Michelin, Belisle et quelques autres.

... un grand nombre de vos Pétitionnaires sont encore des descendants des sus-nommés et mentionnés, dont quelques-uns du côté des femmes, exerçant les différents métiers qu'ils ont appris de leurs pères et... comme eux, espéraient vivre et mourir dans un état qui semblait héréditaire dans leurs familles. ${ }^{2}$
\end{abstract}

\section{RÉSUMÉ}

À travers l'expérience des mouleurs originaires des Forges du Saint-Maurice, ce texte cherche à mieux cerner la participation des Canadiens français à la formation de la classe ouvrière, les

\footnotetext{
1 Cet article constitue une version remaniée de notre communication présentée au congrès de l'Institut d'histoire de l'Amérique française, Trois-Rivières, octobre 1988. Nous tenons à remercier Joanne Burgess, Bettina Bradbury, France Gagnon, Bruno Ramirez, Jean-Claude Robert et Roch Samson, pour leurs judicieux commentaires. Cette recherche a été réalisée grâce à une bourse du Conseil de recherches en sciences humaines du Canada (Ottawa) et une bourse de la Fondation Desjardins (Montréal).

2 Extraits de la pétition adressée à l'Honorable D. Daly, secrétaire provincial, signée par six ouvriers des Forges du Saint-Maurice au nom de l'ensemble des familles de la communauté. Province du Canada, Journaux de l'Assemblée législative (Montréal, Louis Perrault, 1846), 5: 269.
} 
relations entre mobilité géographique et activité syndicale, et le rôle du métier, de la famille et du voisinage dans les mouvements migratoires et l'adaptation des travailleurs à un nouveau milieu.

L'étude des mouleurs dans le village des Forges du Saint-Maurice révèle l'existence de solidarités familiales et communautaires très intenses liant ces travailleurs depuis la fin du XVIIle siècle. Au cours des décennies 1830 et 1840 , les mouleurs mettent en place les fondements d'un réseau communautaire qui s'étend à plusieurs localités du Québec, participant ainsi à l'expansion de l'industrie du moulage à travers la province.

À partir de la fin des années 1850 , dans le contexte du déclin des forges mauriciennes et l'émergence de Montréal comme centre de la sidérurgie québécoise, leur réseau communautaire s'avère un outil essentiel dans leur migration et adaptation au milieu montréalais. Les mouleurs se concentrent graduellement dans la métropole, où ils prennent une part active à différentes actions revendicatives pour défendre ou promouvoir le statut de leur métier. Mais leur ardeur est longtemps tempérée par les tensions ethniques et linguistiques locales.

\begin{abstract}
Through the experience of the moulders coming from the St.Maurice Forges, this paper tries to improve our understanding of the participation of French Canadians to working class formation, the relations between geographical mobility and union activity, and the part played by craft, family and neighbourhood in the migratory movements and adaptation of workers to a new social environment.
\end{abstract}

The study of the moulders at the St.Maurice Forges village reveals the existence of very strong family and community solidarities linking together these workers since the end of the eighteenth century. In the course of the 1830s and 1840s, the moulders put in place the foundations of a community network which extends to many Québec localities, participying, thus, in the spread of the moulding industry throughout the province.

Starting with the end of the 1850s, in a context of decline of the Mauricie forges and the emergence of Montréal as the center of Québec siderurgy, their community network proves to be an essential tool in their migration and adaptation to the Montreal environment. The moulders concentrate gradually in the metropolis, where they take an active part in different actions of protest to defend or promote the status of their craft. But their ardour is for a long time moderated by local ethnic and linguistic tensions.

Peu de renseignements existent sur la vie du mouleur Louis Imbleau. Pourtant, son histoire révèle des éléments importants pour la compréhension du mouvement ouvrier au Québec pendant la phase initiale de l'industrialisation. Louis Imbleau est né aux Forges du SaintMaurice en 1818. Son arrière-grand-père, originaire de Dijon en France, a travaillé comme goujat, puis comme chauffeur, aux Forges sous le Régime français. L'adoption du métier de mouleur par le père de Louis, vers 1800 , instaure dans la famille une tradition de transmission du métier de père en fills ${ }^{3}$.

${ }^{3}$ Le «goujat» est un journalier travaillant sous les ordres des chauffeurs et marteleurs, ces travailleurs de métier aussi appelés "gros forgerons» et chargés de la transformation du fer en gueuse en fer en barre. Les renseignements sur le père et l'arrière-grand-père de Louis Imbleau proviennent des ouvrages suivants: Marie-France Fortier, La structuration sociale du village industriel des Forges du Saint-Maurice: étude quantitative et qualitative (Parcs Canada, 1977), travail inédit no 259, 10, 13 et 178; Luce Vermette, Monographies d'employés aux Forges du Saint-Maurice (Parcs Canada, 1978), travail inédit no 292, 169-173. 
Louis apprend l'art du moulage aux Forges vers le milieu des années 1830. À la fin de son apprentissage, il travaille quelque temps à Vergennes dans l'État du Vermont. De retour au Bas-Canada, il épouse en 1840 Marguerite Laroche, une fille des Forges, et, par la suite, il est employé à cet endroit. Mais, en 1852, on le retrouve à Trois-Rivières où lui et neuf autres mouleurs des Forges constituent la forte majorité des mouleurs de la ville. En 1861, il oeuvre aux Forges Radnor en compagnie de mouleurs dont la plupart sont originaires des Forges du Saint-Maurice. Peu de temps après la fermeture temporaire des Forges Radnor, en 1866, il s'établit à Montréal, comme plusieurs de ses camarades de travail. Sa famille l'accompagne dans tous ces déplacements. En 1869, il devient membre de la section montréalaise de l'Iron Molders International Union. La durée de cette première adhésion au syndicát est indéterminée. Mais, vers 1880, dans un contexte d'expansion rapide des effectifs du syndicat, Louis joint à nouveau l'organisation avec, cette fois, ses deux fils également devenus mouleurs. Il meurt à Montréal en $1890^{4}$.

La tradition familiale du métier et l'itinéraire professionnel de Louis Imbleau comportent de fortes similarités avec ceux de nombreux mouleurs originaires des Forges, qui se concentrent à Montréal à partir de 1857. Ces migrations s'expliquent d'abord par l'élargissement des possibilités d'emploi et le déplacement des centres de production. Mais elles s'organisent à l'intérieur d'une solidarité communautaire qui les conditionne aussi. Cette solidarité communautaire est fondée sur la pratique collective d'un métier et les liens de parenté et de voisinage entre familles de mouleurs formés aux Forges du Saint-Maurice ${ }^{5}$. Dans un contexte de modifications de la demande d'emploi, ces liens de solidarité se déploient à l'échelle provinciale par le biais de filières migratoires. L'objectif semble être la constitution d'un vaste réseau d'entraide qui permette aux membres de cette collectivité l'exercice du métier, la transmission familiale du métier et parfois l'entrée en affai-

\footnotetext{
$4 \quad$ Les renseignements concernant Louis Imbleau sont tirés du Répertoire de mariage de la Cathédrale de Trois-Rivières, 1634-1971; de la liste des ouvriers des Forges de 1842 dressée pour Matthew Bell; des listes nominatives de recensements de 1852, 1861, 1871 et 1881; des Montreal Directory de 1866 à 1881; des rapports de la section 21 de l'Iron Molders International Union dans l'Iron Molders Journal (dorénavant IMJ); et des registres de sépultures de la paroisse NotreDame de Montréal.

5 Thomas Bender résume bien ce que nous entendons par «communauté»: «A community involves a limited number of people in a somewhat restricted social space or network held together by shared understandings and a sense of obligation. Relationships are close, often intimate, and usually face to face.» Il précise: «This network... is the essence of community, and it may or may not be coterminous with a specific, contigous territory.» Thomas Bender, Community and Social Change in America (New Brunswick (New Jersey), Rutgers University Press, 1978), 7. Le concept de «solidarité communautaire», tel que développé dans notre texte, ne fait pas référence à la notion de «marginalité», contrairement à la formulation présentée par Gérard Bouchard dans son article «La dynamique communautaire et l'évolution des sociétés rurales québécoises aux $19 \mathrm{e}$ et $20 \mathrm{e}$ siècle. Construction d'un modèle», Revue d'histoire de l'Amérique française, 40,1, (été 1986): 51-71.
} 
res. Le potentiel perturbateur de ces migrations est de plus amoindri par la transposition et la reproduction en milieux d'accueil de ces relations de parenté et de voisinage.

Dispersés à travers douze localités du Québec en 1861, les mouleurs originaires des Forges du Saint-Maurice constituent 20\% des 315 mouleurs de la province et $38 \%$ des effectifs canadiens-français du métier ${ }^{6}$. Ces mouleurs vont se regrouper graduellement à Montréal pour former $12 \%$ des 319 mouleurs de la ville, en 1871, et représenter $25 \%$ des mouleurs canadiens-français de l'agglomération. Sensibles à la préservation du statut de leur métier, ils occupent une place importante à l'intérieur du corps de métier des mouleurs, membre de la Grande Association des corps de métier de Montréal, et au sein de la section montréalaise de l'Iron Molders International Union.

Ces migrations et ces dispositions prononcées à défendre ou à promouvoir le statut du métier, chez les mouleurs originaires des Forges du Saint-Maurice, s'opposent à cette assertion longtemps avancée que la mobilité géographique élevée des travailleurs agit contre le développement d'une conscience ouvrière ${ }^{7}$. Nous allons voir, au contraire, que, malgré plusieurs migrations, les solidarités de métier et de parenté se maintiennent chez les mouleurs originaires des Forges. Liés par des traditions de métier provenant du même lieu et qui, pour plusieurs d'entre eux, remontent au moins à la la fin du XVIIIe siècle, ces ouvriers démontrent que les liens communautaires de la période pré-industrielle ne s'affaiblissent pas au début de l'industrialisation. Au contraire, ils vont soutenir les travailleurs durant la période initiale de formation de la classe ouvrière et du mouvement ouvrier.

L'analyse des mouleurs originaires des Forges fait également sortir de l'ombre les ouvriers canadiens-français formés pendant la période pré-industrielle et montre le rôle important qu'ils ont joué dans l'émergence du marché du travail capitaliste et du mouvement ouvrier au début de l'industrialisation ${ }^{8}$. On a expliqué ces processus en mettant l'accent essentiellement sur les travailleurs étrangers, surtout les paysans irlandais catholiques et les artisans anglais et écossais protestants ${ }^{9}$.

\footnotetext{
6 Listes nominatives du recensement de 1861 pour la province de Québec.

7 Au Canada, le principal tenant de cette thèse est Michael Katz. Voir son chapitre «Transiency and Social Mobility» dans Michael Katz, The People of Hamilton, Canada West, Family and Class in a Mid-Nineteenth-Century City (Cambridge, Harvard University Press, 1975), en particulier les pages 24,111 et 112 . Katz réaffirme sa position dans son livre Michael Katz, Michael J. Doucet et Mark J. Stern, The Social Organization of Early Industrial Capitalism (Cambridge, Harvard University Press, 1982); voir le chapitre sur la "Transiency», en particulier la page 130. Pour un bilan historiographique de cette thèse et sa remise en question, on peut consulter l'article de Gordon Darroch, «Migrants in the Nineteenth Century: Fugitives or Family in Motion?», Journal of Family History, 6 (Fall 1981): 257-277.

8 Par «mouleur originaire des Forges du Saint-Maurice», nous entendons les mouleurs nés aux Forges ou de parents nés à cet endroit.

9 H. C. Pentland, Labour and Capital in Canada, 1650-1860 (Toronto, James Lorimer \& Co., 1981), 61-95.
} 
Selon cette thèse, les Canadiens français ne se retrouvent sur le marché du travail capitaliste, en proportion significative, qu'à partir de $1870^{10}$. Cette interprétation développée par H. C. Pentland a été reprise par plusieurs historiens des travailleurs ${ }^{11}$.

Notre recherche contribue ainsi à la récente remise en question de la participation des Canadiens français à la constitution de la classe ouvrière. La thèse de doctorat de Joanne Burgess, sur la communauté artisanale du cuir, au cours des années 1790 à 1831 , montre que dorénavant les Canadiens français doivent être perçus comme des acteurs importants au cours des premières décennies de la formation de la classe ouvrière. Les travaux de Marie-France Fortier et de Roch Samson insistent aussi sur le fait que les ouvriers des Forges du Saint-Maurice, majoritairement canadiens-français, contribuent dès la fin du XVIIIe siècle à former une réserve de main-d'oeuvre représentant un facteur important dans l'implantation d'autres forges en Mauricie. Nous avons nous-même montré que les Canadiens français occupent une place importante parmi les mouleurs montréalais et leurs actions revendicatives au cours des années 1859 à 1881 , un secteur présenté jusqu'à maintenant par l'historiographie comme le domaine privilégié des travailleurs d'origine britannique ${ }^{12}$.

Notre étude des mouleurs originaires des Forges du Saint-Maurice fait appel à diverses sources. Le groupe de mouleurs a d'abord été cerné par le dépouillement des listes d'ouvriers des Forges de 1829 et de 1842 et des listes nominatives des recensements de l'établissement de 1831, 1852,1861 et $1871^{13}$. Les listes nominatives du recensement de 1831 pour Trois-Rivières complètent cette étape ${ }^{14}$. Nous avons ensuite suivi

10 H. C. Pentland, «The Development of a Capitalistic Labour Market in Canada», Canadian Journal of Economics and Political Science, 25 (November 1959): 450-461.

11 Jacques Rouillard et Judith Burt, «Le mouvement ouvrier», Jean Hamelin, dir., Les travailleurs québécois (Montréal, Presses de l'Université du Québec, 1975), 110; Fernand Harvey, Révolution industrielle et travailleurs: une enquête sur les rapports entre le capital et le travail au Québec à la fin du XIXe siècle (Montréal, Boréal Express, 1978), 25; Bryan D. Palmer, Working-Class Experience, the Rise and Reconstitution of Canadian Labour, 1800-1980 (Toronto, Butterworth \& Co., 1983), 26.

12 Joanne Burgess, Work, Family and Community: Montreal Leather Craftsmen, 17901831 , thèse de doctorat, département d'histoire, UQAM, 1986, 725 p.; Marie-France Fortier, La structuration sociale..., 38; Roch Samson, "Une industrie avant l'industrialisation: le cas des Forges du Saint-Maurice», Anthropologie et société, 10,1 (1986): 99; Peter Bischoff, «La formation des traditions de solidarité ouvrière chez les mouleurs montréalais: la longue marche vers le syndicalisme (1859-1881)», Labour/Le Travail, 21 (printemps 1988): 9-43.

${ }_{13}$ La liste d'ouvriers datant d'août 1829 se trouve aux Archives nationales du Canada, RG4, A, vol. 225, page 84; la liste du 27 décembre 1842 est publiée dans Province of Canada, Appendix to the 4th volume of the Journals, Appendix «O», 4, 1 (Montréal, 1845). L'ampleur de la recherche nous a amené à considérer seulement les individus dont la profession déclarée dans les recensements et les annuaires est celle de mouleur. Cela peut avoir pour effet de mésestimer le moment exact des migrations et leur ampleur. Mais nous considérons que les résultats obtenus sont quand même très significatifs.

14 Le locataire des Forges possède en effet une fonderie à Trois-Rivières, la seule de cette petite localité. 
le déplacement de ces mouleurs à travers le Québec, en tentant de les repérer premièrement à Montréal, au moyen des recensements de 1831, $1842,1861,1871$ et 1881 et du rôle d'évaluation de $1851^{15}$. Les mouleurs ont été ensuite recherchés dans le reste de la province à travers les listes nominatives des recensements de 1852 et 1861 et à Trois-Rivières au recensement de 1871 .

Bien sûr, ces sources avec leur coupe décennale ne permettent pas de dater avec précision les mouvements migratoires des mouleurs. C'est pourquoi il a fallu consulter une série d'annuaires: les annuaires du Canada de 1851, 1853, 1857-1858, 1864-1865 et 1871; les annuaires de Québec de 1855, 1857, 1861-1862, 1863-1864, 1872-1873, 1876$1877,1878-1879$ et 1882-1883; les annuaires de Trois-Rivières de 1876, 1879,1880 et 1884; et les annuaires de Montréal de 1842 à 1881 . De plus, l'étude des migrations de mouleurs vers Montréal pendant la première moitié du XIXe siècle a été complétée par le dépouillement des registres de baptême et de mariage de la paroisse Notre-Dame de Montréal (grâce aux index existants) pour les années 1800 à 1850.

Nous avons ensuite établi les liens de parenté entre les mouleurs originaires des Forges. Les répertoires de mariage et les registres d'état civil des paroisses des comtés de Saint-Maurice, de Champlain, de Joliette, et des cités de Montréal, Québec et Trois-Rivières se sont révélés d'une grande importance lors de cette démarche. Les informations réunies ont été complétées grâce au fichier Loiselle ${ }^{16}$. Ces sources ont permis d'établir dans un premier temps l'ascendance sur trois générations et plus de la majeure partie des mouleurs et, dans un deuxième temps, les liens de parenté complexes qui unissent ces ouvriers.

Enfin, les actions revendicatives des mouleurs originaires des Forges du Saint-Maurice ont été étudiées principalement à travers deux sources. Leur participation à la section 21 de l'Iron Molders International Union est saisie par l'entremise des rapports mensuels de la section au journal de l'organisation, l'Iron Molders Journal, durant les années 1864 à 1881 . Un autre journal, L'Union nationale, permet également d'analyser l'adhésion des mouleurs à l'éphémère Grande Association des corps de métier de Montréal, en 1867.

Les résultats de notre recherche sont présentés en deux parties. Nous verrons d'abord que les Forges ont contribué par la formation d'ouvriers mouleurs largement apparentés entre eux, à produire une main-d'oeuvre qualifiée autochtone. Cette dernière connaît, dès la pre-

\footnotetext{
15 Nous remercions le Groupe de recherche sur l'histoire de Montréal (UQAM) pour nous avoir fourni la liste des mouleurs montréalais des recensements de 1831 et 1842 et du rôle d'évaluation de 1851. Le rôle d'évaluation de 1851 est utilisé car le recensement de 1851-1852 a été détruit ou perdu.

16 Antonin Loiselle (père), Fichier Loiselle des mariages.
} 
mière moitié du XIXe siècle, des migrations vers d'autres localités qui préparent le terrain aux grandes migrations de la période suivante. En second lieu, on expliquera comment, en raison du déplacement des centres de production, les mouleurs originaires des Forges se concentrent graduellement à Montréal, par des migrations en chaîne, et le rôle influent qu'ils occupent au sein des actions revendicatives des mouleurs montréalais.

\section{1 - APOGÉE ET DÉCLIN DU MOULAGE AUX FORGES, 1829-1858}

\section{$A$ - Les mouleurs aux Forges en 1829}

Au début de la période, le métier est contrôlé aux Forges par une oligarchie. Composé d'un nombre très restreint de familles apparentées, ce groupe s'est constitué à partir d'un processus de transmission familiale du métier et d'alliances matrimoniales s'étendant sur une période relativement longue.

Les Forges du Saint-Maurice sont en exploitation depuis 1737. Situées sur la rive droite de la rivière Saint-Maurice, à environ huit milles de son embouchure, elles abritent une communauté retirée, accessible par terre et par eau à partir de Trois-Rivières. L'importance de cette agglomération villageoise est très récente. De 1784 à 1829, la population des Forges, majoritairement française, passe de 149 habitants à 395 et, après 1830 , elle se stabilise autour de 400 personnes. En effet, engageant presqu'exclusivement au XVIIIe siècle des ouvriers spécialisés, dont le travail était requis sur le site même, les Forges deviennent, sous l'administration de Matthew Bell ${ }^{17}$, un village qui intègre au début du XIXe siècle, la plupart des ouvriers requis en amont et en aval des opérations de fonte ${ }^{18}$. La physionomie de l'agglomération change donc grandement avant 1829 , pour se modifier probablement beaucoup plus lentement par la suite. On peut donc penser que le plan schématique des Forges du Saint-Maurice daté de janvier 1845 (figure 1) expose assez fidèlement l'apparence qu'acquiert le village vers la fin des années 1820.

La réduction du minerai de fer et la fabrication d'objets en fonte, effectuées au haut-fourneau et à la moulerie, constituent l'activité la plus importante du village. Plusieurs techniques de moulage sont utilisées. Quel que soit le procédé utilisé, le moulage exige généralement des connaissances techniques considérables, car les opérations apparemment simples demandent un long entraînement (quatre années d'apprentissage environ) et plusieurs années d'expérience ${ }^{19}$. Vers la fin des

17 Matthew Bell est locataire des Forges de 1793 à 1846.

18 Roch Samson, Les ouvriers des Forges du Saint-Maurice: aspects démographiques (17621851) (Parcs Canada, 1983), 89-104, travail inédit.

19 Pour une description détaillée du métier, le lecteur est référé à Peter Bischoff, Les ouvriers mouleurs à Montréal, 1859-1881, mémoire de maîtrise, département d'histoire, UQAM, 1986, 47-56. 
FIGURE 1

Plan schématique des Forges du Saint-Maurice, janvier 1845.

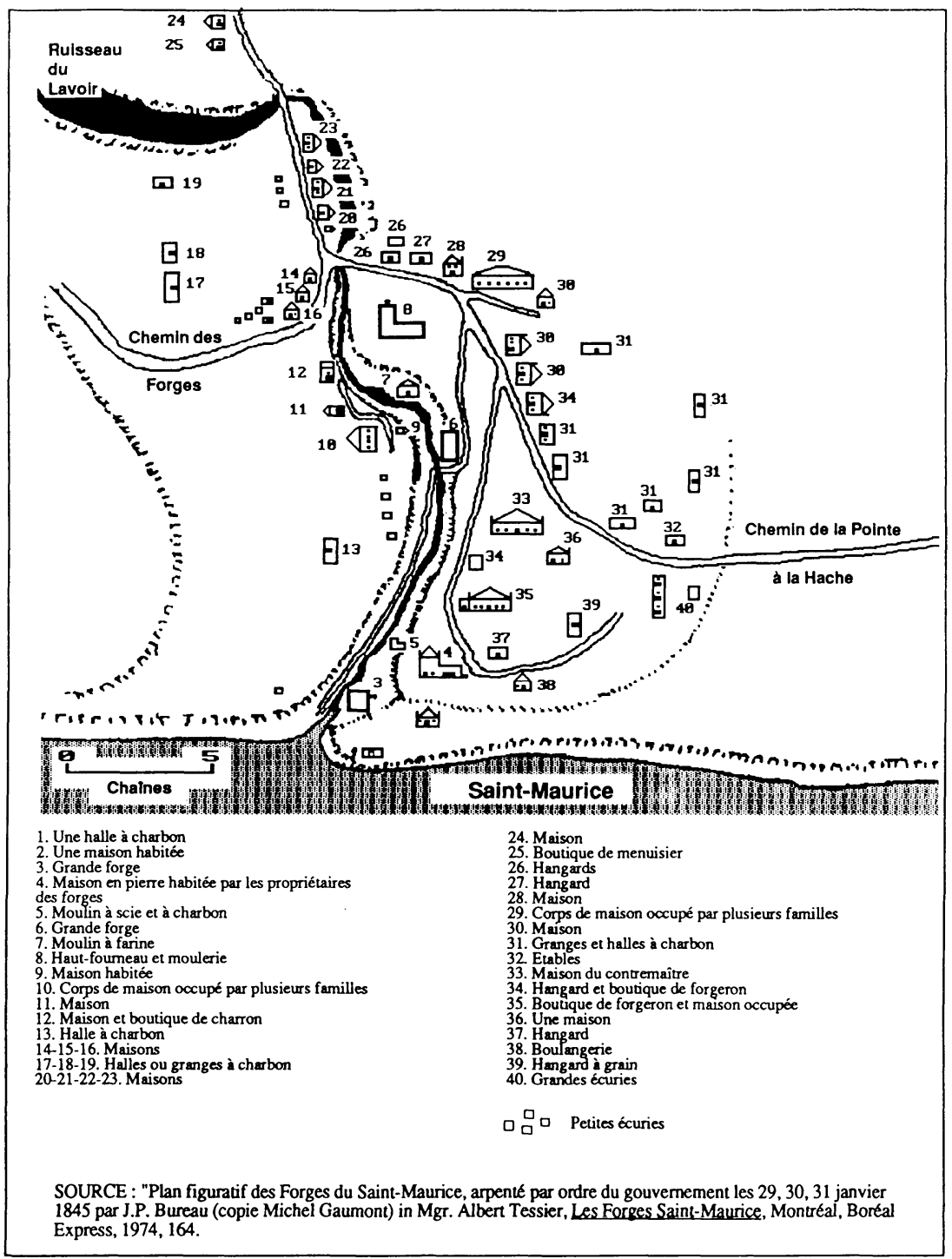

années 1820, on coule aux Forges deux tonnes et quart par jour de plaques de poêles, de chaudières, d'équipement de moulin, de matériel aratoire, etc. ${ }^{20}$.

20 Lieutenant Baddely, «Essay on the Localities of Metallic Minerals in the Canadas, with some Notices of their Geological Associations and Situation, \&c», Transactions of the Literary 
C'est à partir de la fin du XVIIIe siècle que l'entreprise a de plus en plus recours au phénomène de transmission familiale de différents métiers pour répondre à ses besoins en main-d'oeuvre. À ce moment se forme ce qu'on pourrait presque appeler des «clans»: père, fils, beaupère, beaux-frères, oncles, neveux, cousins, etc., exercent le même métier, et ce, pendant des générations successives ${ }^{21}$.

La composition du groupe des neuf mouleurs oeuvrant aux Forges du Saint-Maurice et des cinq engagés à la fonderie de Bell à TroisRivières en 1829 , montre que ce métier a connu une telle évolution. Depuis la fin du XVIIIe siècle, les pères ont eu fortement tendance à initier un fils ou plus à l'art du moulage. Non seulement ont-ils bénéficié de cette manière d'un soutien au travail, mais ils ont profité aussi de revenus supplémentaires pour leur famille et ont préparé leurs fils à la vie adulte ${ }^{22}$. En 1829, le métier de mouleur, comme ceux de gros forgeron et de forgeron ${ }^{23}$, est aux mains de quelques familles installées depuis plusieurs générations aux Forges. Ces familles de mouleurs sont unies par un réseau complexe de liens de parenté gravitant autour des familles «Terreau» (voir figure 2$)^{24}$. Leurs ancêtres étaient des travailleurs de métier qui arrivèrent de France sous le Régime français ou qui s'installèrent aux Forges au cours des premières décennies après la Conquête $^{25}$. Les familles «Slicer» semblent à première vue indépendantes de ce réseau de parenté, mais nous croyons qu'il en est autrement: le père de John et de Robert, le premier de la lignée des «Slicer», a en effet épousé une Josephte Mailloux, fort probablement une parente des mouleurs Joseph et Pierre Mailloux ${ }^{26}$.

La formation d'une oligarchie chez les mouleurs s'explique apparemment par la nature même du marché du travail dans la sidérurgie. Ce dernier, peu développé, ne permet pas à l'entreprise de puiser librement dans une réserve de main-d'oeuvre. Dans ces conditions, l'établissement se replie sur l'institution familiale qui assure la reproduction

and Historical Society of Quebec (Québec, Thomas Cary \& Co., 1831), II: 401; Benjamin Sulte, dans Gérard Malchelosse, éd., Les forges Saint-Maurice (Montréal, G. Ducharme, 1920), Mélanges historiques, 6: 179.

21 Marie-France Fortier, La structuration sociale..., 21.

22 Une telle stratégie est également employée par les artisans canadiens-français du cuir à Montréal, à la même époque. Voir Joanne Burgess, op. cit., section «Fathers and Sons», 268274.

23 Roch Samson, Les ouvriers des Forges..., 95.

24 Nous remercions Roch Samson, du Service canadien des parcs à Québec, pour nous avoir fourni une copie de la liste des ouvriers des Forges du Saint-Maurice datée du mois d'août 1829.

is Nous avons utilisé les répertoires de mariage et avons complété nos informations au moyen des travaux suivants: Luce Vermette, Monographies d'employés..., 155-162, 230-236; Marie-France Fortier, La stucturation sociale ..., tableau 4; Benjamin Sulte, dans Gérard Malchelosse, éd., Les Forges Saint-Maurice, 109, 112 et 169.

Le mariage a été célébré à l'église protestante de Trois-Rivières, le 14 juillet 1771 . Le registre de mariage n'indique malheureusement pas le nom des parents des mariés, ce qui nous empêche d'établir avec certitude le lien de parenté avec le reste du réseau des mouleurs en 1829. 
FIGURE 2

Liens de parenté entre les mouleurs originaires des Forges du Saint-Maurice. Août 1829.

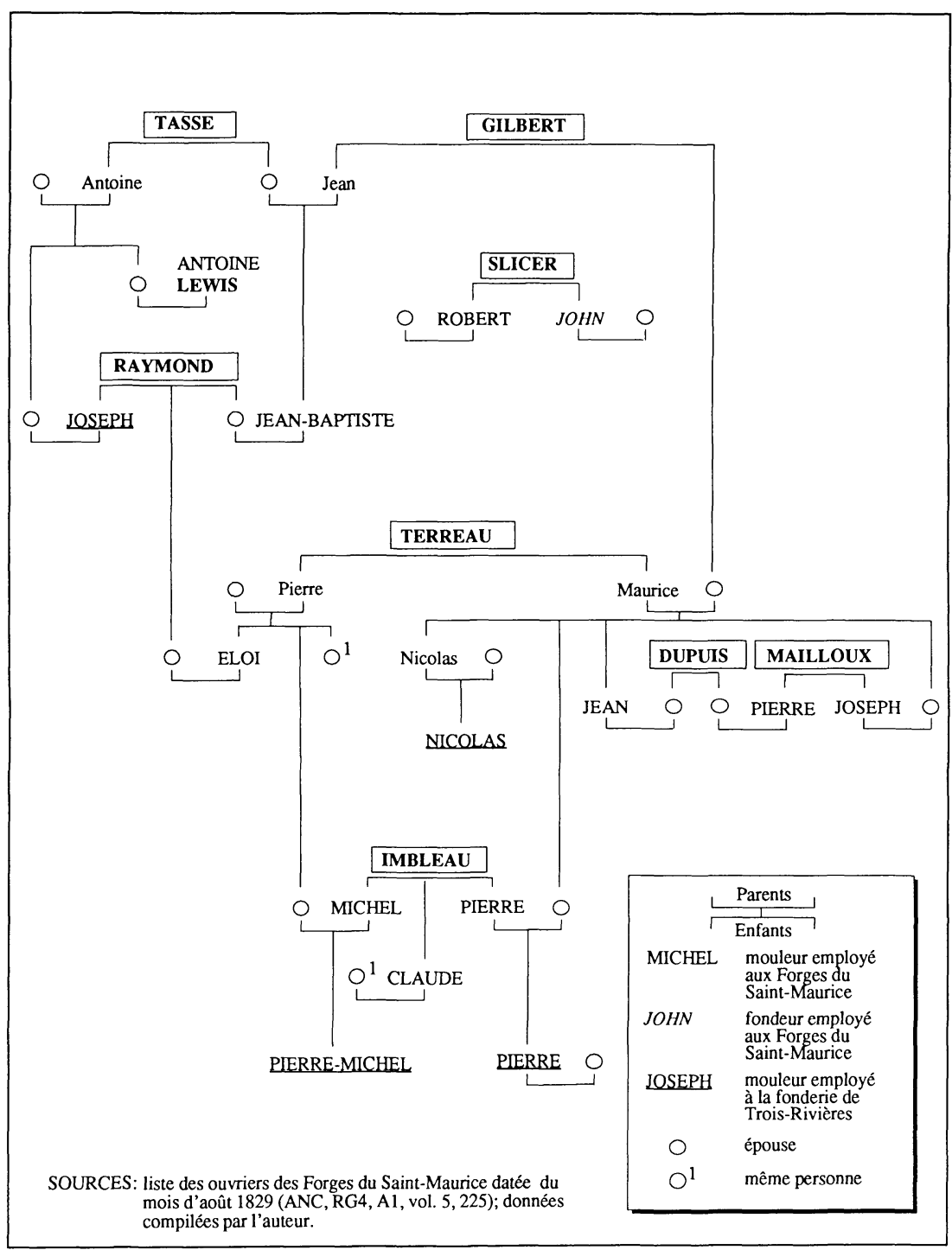

de la main-d'oeuvre et la transmission du savoir-faire indispensable à son fonctionnement ${ }^{27}$ (voir figure 3 ). Ces familles, qui se côtoient

27 Roch Samson, Les ouvriers des Forges..., 81. 
continuellement au travail et dont le marché matrimonial est restreint vu l'isolement des Forges, forment alors un réseau de parenté très serré ${ }^{28}$.

\section{$B$ - Les premières filières migratoires et la formation d'un réseau de parenté interrégional}

Durant les années 1830 et 1840 , les effectifs de mouleurs prennent une forte expansion aux Forges. Cette période marque la fin de la position oligarchique des anciennes familles dans le métier. Ces dernières mettent en place les premières filières migratoires qui amènent probablement un échange d'information et encouragent la mobilité géographique des mouleurs sur une aire de plus en plus vaste. Ces premières migrations en chaîne et migrations circulaires, encore de dimensions modestes, préparent le terrain aux grandes migrations prenant place entre 1858 et 1867.

Sous les administrations successives de Matthew Bell, d'Henry Stuart et de James Ferrier, durant les années 1830 et 1840, la part occupée par les produits de fonte, surtout les poêles, dans la production totale de l'établissement, augmente ${ }^{29}$. Les effectifs de mouleurs passent de neuf, en 1829 , à vingt-six, en $1852^{30}$. On pourrait croire que, dans ce contexte d'augmentation des effectifs, les familles de mouleurs, déjà en place en 1829, vont conserver leur mainmise sur le métier en investissant les nouveaux postes. Mais Roch Samson montre qu'il n'en est rien. Des sept noms patronymiques de mouleurs en 1829, seulement cinq sont toujours représentés en 1852. L'augmentation des effectifs traduit en fait l'introduction de nouvelles familles au sein du métier: neuf nouveaux noms patronymiques apparaissent, la plupart représentant des familles d'ouvriers apprêteurs et transporteurs qui se sont établies aux Forges au cours des premières décennies du XIXe siècle ${ }^{31}$. Cependant, nos recherches indiquent que ces nouvelles familles sont, entre-temps, intégrées au sein des alliances matrimoniales des familles de mouleurs plus anciennes.

${ }^{28}$ En Franche-Comté, d'où sont originaires une bonne partie des ouvriers métallurgistes des Forges du Saint-Maurice, les travailleurs de la métallurgie utilisent, au cours des décennies 1770-1820, des stratégies quasi identiques à celles des mouleurs des Forges du Saint-Maurice: transmission familiale du métier et alliances matrimoniales au sein d'un même métier. Cette similarité s'expliquerait-elle par l'influence d'un héritage artisanal qui, plongeant ses racines dans la sidérurgie française ancienne, pourrait amener des pratiques de métier analogues? Voir Denis Woronoff, L'industrie sidérurgique en France pendant la révolution et l'empire (Paris, l'École des hautes études en sciences sociales, 1984), 160-162.

${ }_{29}$ Michel Bédard, Le contexte de fermeture des Forges du Saint-Maurice (Parcs Canada, 1980), 15-36, travail inédit.

30 Deux des mouleurs recensés en 1852, Joseph Boisvert et Julien Rivard, sont âgés respectivement de 87 ans et de 73 ans. Il serait très étonnant qu'ils pratiquent encore ce métier éreintant. Les effectifs réels se résument probablement à 24 ouvriers.

${ }_{31}$ Les «Slicer» et «Raymond» ont disparu des Forges en 1852. Roch Samson, Les ouvriers des Forges..., tableau 44, «Le devenir des familles selon leur apport comparé d'ouvriers entre 1829 et $1851 »$. 
FIGURE 3

Transmission familiale du métier de mouleur au sein de la famille Imbleau.

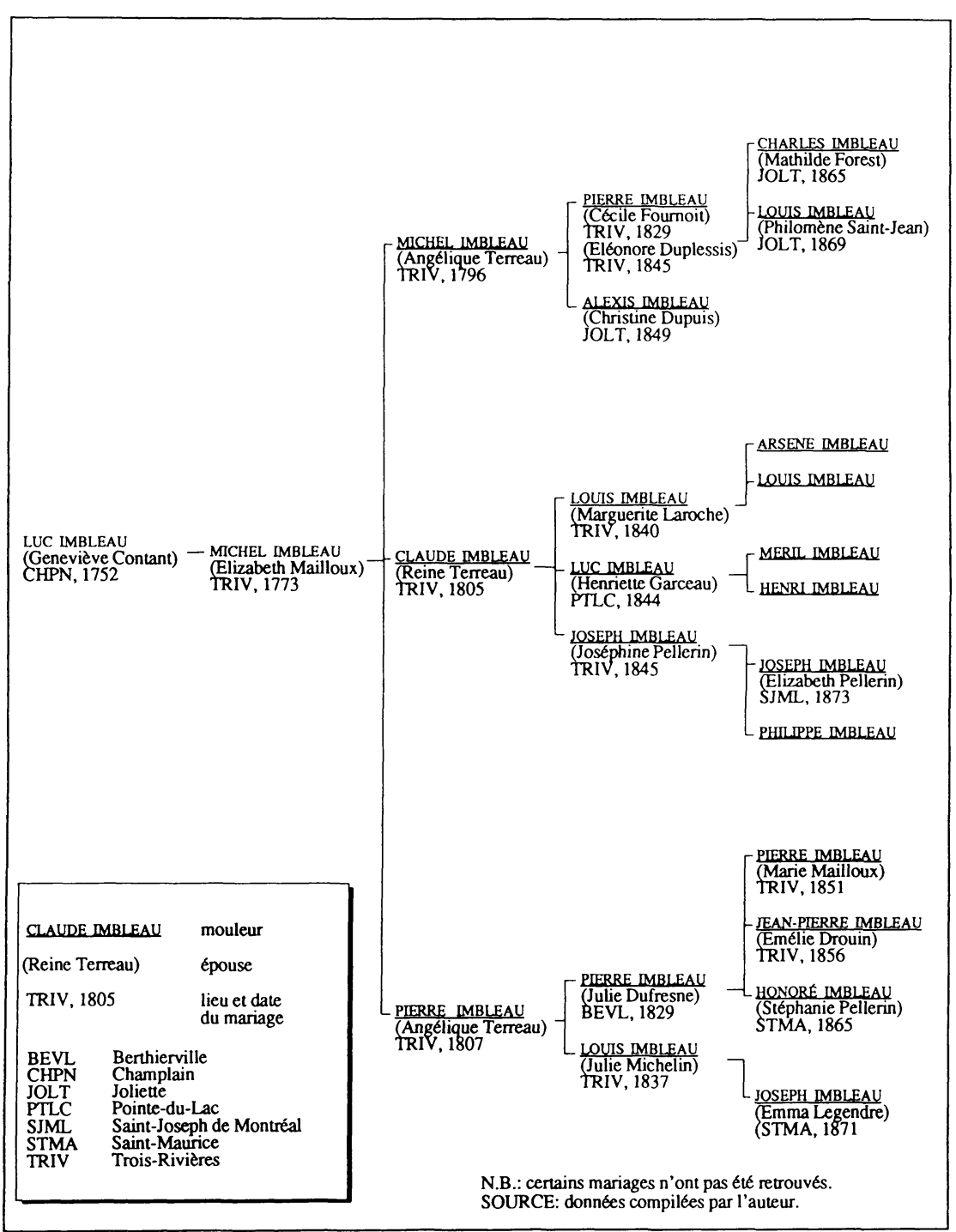

Au cours des décennies 1830 et 1840 , la baisse en importance des familles occupant le métier de mouleur en 1829, tient en bonne partie à la migration de leurs membres vers d'autres centres industriels. On peut alors se demander si l'entrée de nouvelles familles dans le métier 
pendant cette période, résulte du départ volontaire de familles membres de l'oligarchie ou, au contraire, d'une tactique de la direction de l'entreprise qui tient à atténuer le contrôle de ces familles sur la transmission du métier, entraînant de ce fait le départ de plusieurs de ses éléments. Le manque d'information sur cette période empêche toute certitude à ce sujet ${ }^{32}$.

Le départ des anciennes familles de mouleur remonte aux années 1830. Vers 1836-1837, une première filière migratoire de mouleurs des Forges se forme en direction de Montréal: ce n'est pas une migration d'individus, mais celle de plusieurs noyaux familiaux apparentés gravitant autour de la famille de Robert Slicer. Montréal n'est pas un milieu tout à fait inconnu pour ce dernier. Fils de John Slicer, un mouleur d'Angleterre venu travailler aux Forges vers 1771, et de Josephte Mailloux, Robert est employé aux Forges de Sainte-Geneviève-de-Batiscan au début du XIXe siècle où il marie une fille de la paroisse. Après la fermeture de cet établissement, Robert travaille à Montréal de 1817 à 1823 au moins, et plusieurs de ses enfants naissent dans cette ville. De 1829 à 1835, il met son art au service des Forges du Saint-Maurice comme l'a fait son père. Il retourne avec sa famille à Montréal vers 1836-1837. Les familles des mouleurs Louis-Théophile Aubry, son gendre, et de Joseph Slicer, son fils, semblent l'accompagner, car elles apparaissent à Montréal à la même période. À partir des années 1840, trois autres fils de Robert s'ajoutent à ce noyau: Alexandre, John et William, tous devenus mouleurs entre-temps, et qui prennent épouse à la ville ${ }^{33}$.

D'autres familles de mouleurs apparentées en provenance des Forges se greffent aussi temporairement à ce groupe: il y a d'abord celles de Pierre Terreau, beau-frère de Joseph Slicer, et de son cousin Joseph Terreau, qui s'installent à Montréal au début des années 1840 . La famille de Thomas Mailloux est également présente en $1843^{34}$. Il est

${ }^{32}$ À un niveau d'ensemble, l'étude démographique de la population des Forges réalisée par Roch Samson souligne une migration continuelle de jeunes hommes de la communauté en raison du manque d'emplois qui les empêche de s'établir. Roch Samson, Les ouvriers des Forges..., 57 62.

33 Jusqu'au début des années 1840 , les fonderies montréalaises, peu nombreuses, produisent surtout des machines à vapeur pour le transport maritime. Leur production se diversifie par la suite dans le matériel roulant de chemin de fer, la machinerie industrielle et agricole, les articles de consommation, etc. Gerald J. J. Tulchinsky, The River Barons, Montreal Businessmen and the Growth of Industry and Transportation, 1837-1853 (Toronto, University of Toronto Press, 1977), 210-218.

34 Les itinéraires de tous ces mouleurs ont été retracés à partir des registres de baptêmes de la paroisse de Notre-Dame de Montréal pour les années 1800 à 1850 et ceux de la paroisse de Trois-Rivières pour les années 1815 à 1820 et 1832 à 1840 . Le choix du parrain et de la marraine des enfants nés à Montréal se fait la plupart du temps au sein de la parenté présente en milieu d'accueil, montrant ainsi le maintien de la sociabilité entre parents après la migration. Ces résultats sont similaires à ceux de l'étude de France Gagnon, Le rôle de la famille dans l'adaptation des migrants de la plaine de Montréal au milieu montréalais: 1845-1875, mémoire de maîtrise, département d'histoire, UQAM, 1986, 133-144. 
intéressant de noter que les deux dernières familles, celles de Joseph Terreau et de Thomas Mailloux, réapparaissent au recensement des Forges du Saint-Maurice, en 1861, pour revenir par la suite à Montréal ${ }^{35}$.

Ces premières migrations laissent croire que, dès les années 1830 , les possibilités d'emploi pour les mouleurs commencent à s'élargir dans la province. Au cours des années 1840, les itinéraires migratoires des mouleurs originaires des Forges touchent d'autres localités indiquant que la situation continue d'évoluer en ce sens. Quelques familles «Terreau» s'installent à Québec et des familles «Imbleau» s'établissent à Joliette, et, dans chaque lieu, l'une d'entre elles ouvre, vers 1846-1847, une fonderie artisanale. Les nombreux mariages de «Terreau» à la paroisse de Saint-Roch de Québec illustrent bien ce phénomène de dispersion des familles de mouleurs (figure 4). Trois-Rivières demeure cependant, hors des Forges, le principal lieu de travail des mouleurs. En 1852, neuf familles de mouleurs y travaillent et deux d'entre elles possèdent chacune une petite fonderie.

Les années 1850 marquent la fin des opérations de moulage sur une grande échelle aux Forges. Au cours de cette période, l'établissement rencontre des difficultés croissantes dans la commercialisation de son principal produit, les poêles, sur ses marchés principaux, les villes de Québec et de Montréal. La compétition américaine lui nuit beaucoup $^{36}$. Mais l'essor des fonderies dans ces villes à la même période doit aussi concourir aux difficultés de mise en marché. Les propriétaires réajustent alors leur production, entre 1853 et 1857, en transformant la forge haute en fonderie de roues de trains. Les mouleurs affectés à la production de ces roues doivent alors maîtriser une technique de moulage à coquille, très récente au Québec, située à la fine pointe de l'industrie du moulage nord-américaine ${ }^{37}$. Malgré cette tentative de s'adapter aux nouvelles conditions du marché, l'établissement doit arrêter ses activités vers 1858 en raison de difficultés financières, de poursuites judiciaires et des pressions déflationnistes des années $1857-1859^{38}$. La fermeture des Forges va durer plusieurs années et entraîner un exode des mouleurs.

\footnotetext{
35 Listes nominatives du recensement des Forges du Saint-Maurice de 1861; sépulture de Joseph Terreau, époux de Marguerite Gauthier, mort à 55 ans, paroisse Notre-Dame de Montréal, 1869, folio 55; Montreal Directory de 1875-1876 et de 1878-1879.

36 The Canadian Merchants' Magazine and Commercial Review, 3,2 (mai 1858): 102 et 104.

37 Je remercie Benoît Gauthier, de l'UQTR, d'avoir porté à ma connaissance la spécificité et la nouveauté de cette technique au Québec.

38 Michel Bédard, Le contexte de fermeture..., 100.
} 
FIGURE 4

Transmission familiale du métier de mouleur au sein de la famille Terreau.

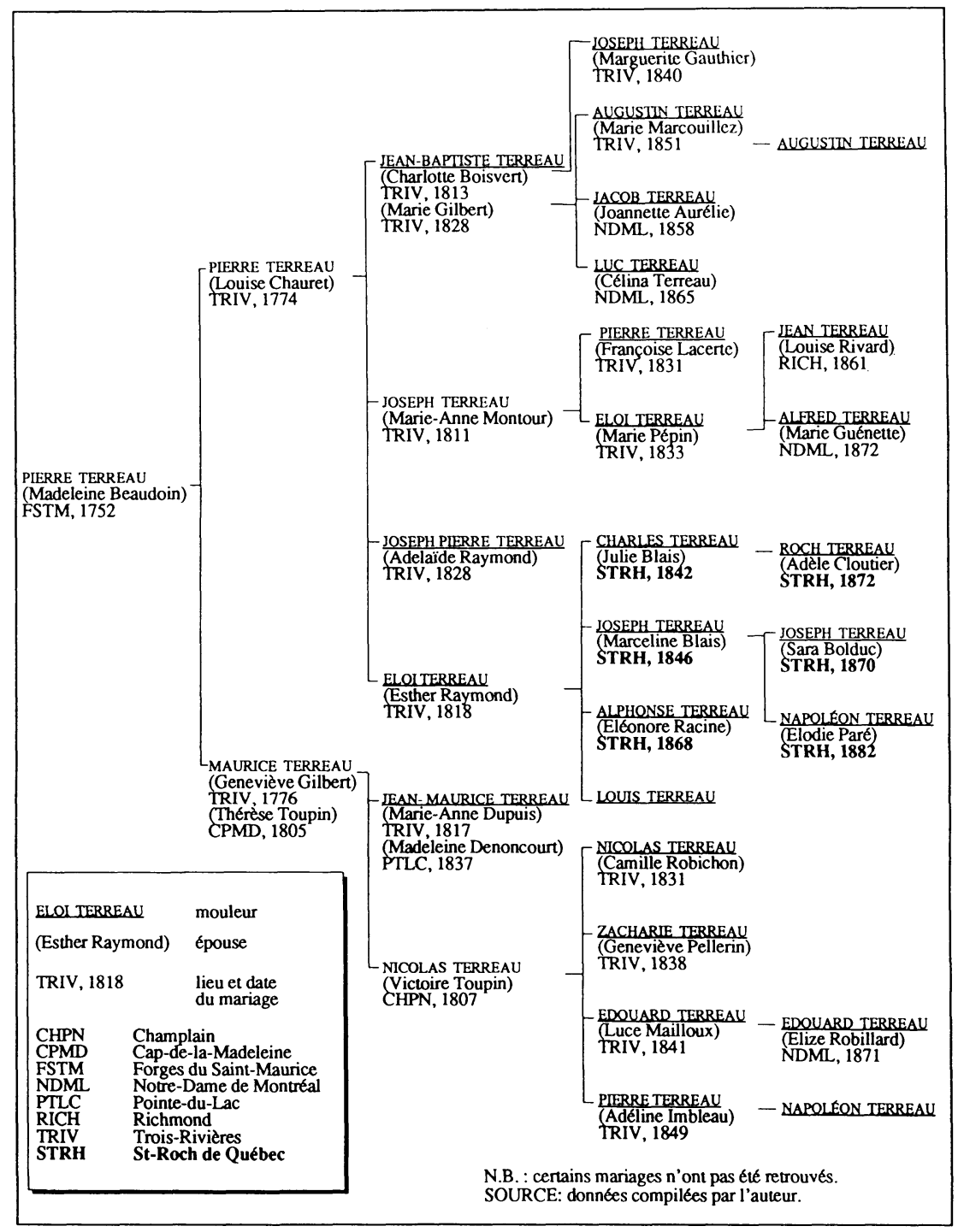




\section{2 - BOULEVERSEMENTS SOCIO-ECONOMIQUES ET CONCENTRATION DES MOULEURS À MONTREAL, 1858-1881}

Au cours des décennies 1830 et 1840 , les mouleurs originaires des Forges du Saint-Maurice, presqu'essentiellement ceux de l'ancienne oligarchie, mettent en place les fondements d'un réseau communautaire, où les liens de parenté sont prépondérants, et qui s'étend à plusieurs localités du Québec. À partir des années 1850 , ce réseau va prendre une importance nouvelle, car, à deux reprises, les mouleurs originaires des Forges sont forcés de migrer en masse. Dans un contexte de fermeture des ateliers de moulage dans les villages de forges de la Mauricie, les solidarités communautaires se maintiennent et canalisent progressivement les migrants vers le milieu montréalais, où la demande de mouleurs est en pleine expansion. Leur réseau communautaire s'avère un outil essentiel dans leur adaptation au milieu d'accueil. Entrant dans un cadre industriel où les rapports de production capitalistes sont beaucoup plus développés qu'ailleurs en province, les mouleurs originaires des Forges du Saint-Maurice prennent très tôt une part active à différentes actions revendicatives pour défendre ou promouvoir le statut de leur métier.

\section{A - Déplacement des centres de production et migration des mouleurs}

La cessation temporaire des activités aux Forges, vers 1858, amorce la première migration massive de mouleurs. En 1861, leurs principaux lieux de concentration sont en ordre d'importance: Trois-Rivières, les Forges Radnor et Montréal. À Trois-Rivières, plusieurs d'entre eux travaillent probablement dans deux fonderies, propriétés de mouleurs apparentés, également originaires des Forges du Saint-Maurice: celle de Louis Dupuis qui engage vingt-quatre travailleurs et produit 2500 poêles par année ou celle de Pierre Mailloux, beaucoup plus modeste, n'employant que trois hommes ${ }^{39}$. Bon nombre de mouleurs originaires des Forges du Saint-Maurice oeuvrent également aux Forges Radnor (fondées en 1854) où leurs connaissances de la fabrication de roues de wagons sont probablement très prisées, permettant en fait à cet établissement de produire des roues sur une grande échelle ${ }^{40}$.

Durant les années 1860, une partie importante de ces mouleurs sont forcés de se déplacer à nouveau. Les Forges Radnor font faillite en $1866^{41}$. De plus, les Forges du Saint-Maurice, rachetées par John

\footnotetext{
39 Listes nominatives de recensement de 1861 pour la cité de Trois-Rivières, quartier StLouis, 437 et 588 .

40 Quatorze des dix-sept mouleurs présents aux Forges Radnor, selon le recensement de 1861 , sont originaires des Forges du Saint-Maurice.

41 Les Forges Radnor semblent alors inactives jusqu'en 1873. Voir Benoît Gauthier, Les sites sidérurgiques en Mauricie (Radnor, Saint-Tite, L'Islet), 1983, 22-28, travail inédit; ClaireAndrée Fortin et Benoît Gauthier, dans René Hardy, dir., Description des techniques et analyse du déclin de la sidérurgie mauricienne, 1846-1910 (Trois-Rivières, Centre de recherche en études québécoises, UQTR, février 1988), 126.
} 
MacDougall (de Trois-Rivières), s'orientent dès 1865 vers la production quasi exclusive de fer en gueuse qu'elles vendent sous contrat à l'entreprise sidérurgique de John McDougall \& Co. de Montréal ${ }^{42}$. Ces événements amènent une nette concentration des mouleurs originaires des Forges du Saint-Maurice au principal centre de la sidérurgie québécoise, Montréal ${ }^{43}$.

Certains mouleurs atteignent Montréal dès 1857. Au nombre de quatre chefs de familles, ils vont habiter dans les quartiers de SainteAnne et de Saint-Antoine où ils joignent William et John Slicer, déjà établis depuis les années 1840 . D'autres mouleurs s'y ajoutent au cours des années suivantes. On voit apparaître à partir de 1860 d'étroites relations de voisinage entre ces ouvriers de métier. Ce voisinage devient encore plus évident à mesure que d'autres mouleurs s'installent progressivement. La filière migratoire s'active particulièrement à partir de 1866, lorsque plusieurs autres mouleurs, tant des Forges Radnor que de Trois-Rivières, s'établissent à Montréal. Les déplacements de ces travailleurs, il faut le préciser, constituent surtout des migrations de familles $^{44}$. Au recensement de 1871 , trente-neuf ouvriers mouleurs originaires des Forges sont installés à Montréal, ce qui représente $12 \%$ de la main-d'oeuvre du métier et $25 \%$ des Canadiens français.

Ces mouleurs maîtrisent plusieurs domaines du moulage d'objets en fonte ${ }^{45}$ : les plus importants sont les poêles, le matériel aratoire, les articles creux et les roues de trains ${ }^{46}$. La production d'articles en fonte est un champ en plein développement à Montréal: les fonderies spécialisées dans cet alliage se multiplient et augmentent leur capacité de

\footnotetext{
42 Vers 1872 ou 1873, les Forges du Saint-Maurice abandonnent définitivement les opérations de moulage sur leur site et se consacrent exclusivement à la vente de leur fer en gueuse. Michel Bédard, Le contexte de fermeture..., 109, 125 et 127.

43 Recensements industriels imprimés du Canada de 1861 et 1871.

44 En 1861, six des onze mouleurs originaires des Forges du Saint-Maurice sont des chefs de famille dont le mariage précède leur arrivée à Montréal; trois sont des chefs de famille qui se sont mariés en ville, mais ils y sont venus comme célibataires avec leurs parents. Dix ans plus tard, $50 \%$ des quarante-deux mouleurs originaires des Forges du Saint-Maurice ont contracté mariage avant de s'établir à Montréal; 14\% se sont mariés dans la ville mais semblent avoir migré préalablement avec leurs parents; seulement $7 \%$ se sont mariés à Montréal alors que leurs parents habitent à l'extérieur de la localité; $19 \%$ sont célibataires et vivent dans leur famille; enfin, pour $10 \%$ des mouleurs, tous mariés, nous n'avons pas été en mesure de déterminer la date et le lieu du mariage. Pour une couverture plus exacte, les données des recensements de 1861 et 1871 ont été complétées avec les annuaires de Montréal de 1861-1862 et 1871-1872. La prédominance d'une migration de familles chez les Canadiens français se déplaçant vers Montréal a également été largement démontrée dans l'étude de France Gagnon, Le rôle de la famille..., 58-73.

45 Nous soulignons le fait car il n'est pas évident que ces mouleurs fabriquent des objets en d'autres alliages. Les sources disponibles indiquent qu'ils ont travaillé auparavant dans des établissements spécialisés dans les articles en fonte. Les treize fonderies qu'ils dirigent entre 1846 et 1881 n'emploient apparemment que de la fonte. Il est donc peu probable, mais non impossible, qu'ils oeuvrent dans les nombreuses fonderies montréalaises spécialisées dans le moulage d'objets de bronze.

46 Voir à ce sujet le «Tableau général des produits fabriqués aux Forges du Saint-Maurice, 1732-1883», dans Michel Bédard, Le contexte de fermeture.... 145.
} 
production. Mais dans quels établissements les mouleurs originaires des Forges du Saint-Maurice sont-ils employés? Se regroupent-ils dans certaines fonderies plutôt que dans d'autres?

On peut envisager qu'une partie des mouleurs ayant travaillé à la fabrication de roues de trains, aux Forges du Saint-Maurice et à Radnor, ont continué de suivre le déplacement de cette activité de transformation lorsqu'elle se relocalise à Montréal, et travaillent à la fonderie de roues de wagons de John McDougall \& Co., ouverte vers 1865 près de l'écluse Saint-Gabriel. En effet, les mouleurs originaires des Forges du SaintMaurice qui étaient employés des Forges Radnor en 1861 et qui sont venus s'établir à Montréal, vivent tous à proximité de la fonderie de roues de John McDougall \& Co. en 1871: Pierre Terreau, Louis Pellerin, Pierre Imbleau et Louis Imbleau habitent sur les rues Saint-Martin et Seigneur, à un quart de mille de l'établissement; tandis que Pierre et Antoine Lewis sont établis sur la rue Saint-Félix à environ deux tiers de mille de la fonderie (voir figure 5). Les possibilités qu'ils travaillent chez John McDougall \& Co. sont élevées, car cet établissement de soixante travailleurs représente la seule fonderie spécialisée dans le domaine en $1871^{47}$.

Plusieurs mouleurs sont peut-être également employés dans la plus importante fonderie mise sur pied par leur réseau de parenté au Québec: celle des mouleurs Augustin Terreau ${ }^{48}$, Emmanuel Day $^{49}$ et l'ex-sculpteur Alfred Deblois qui agit en tant que comptable ${ }^{50}$. Située sur la rue Sainte-Anne, dans le quartier du même nom, l'entreprise a été établie en 1870 (tableau 1) ${ }^{51}$. D'abord locataires de l'établissement, les associés en deviennent propriétaires au cours de la décennie $1870^{52}$. Dès

47 Le chiffre est tiré de Ève Martel, L'industrie à Montréal en 1871, mémoire de maîtrise, département d'histoire, UQAM, 1976, 92-93.

${ }_{48}$ Augustin Terreau a déjà dirigé une fonderie à Montréal, en 1857, avec Jacques Marcotte. Mais cette association a été très éphémère. La Patrie, 28 août 1857.

49 Né en 1829 à Saint-Roch (Québec), Emmanuel Day a marié la demi-soeur d'Augustin Terreau au même endroit en 1848 (d'après les registres d'état civil de la paroisse Saint-Roch). Selon les souvenirs de son petit-fils, il a travaillé par la suite quelques années aux Forges du SaintMaurice avant de s'établir à Montréal. Les souvenirs d'Edgar Day nous ont été fournis par Robert Comeau, du département d'histoire de l'UQAM, son petit-fils. Nous l'en remercions. Edgar Day, «Souvenirs», document manuscrit, sans lieu, sans date, 3 pages.

50 Deblois a marié la fille du mouleur Eloi Terreau, cousin d'Augustin Terreau. Registres de mariages de la paroisse Notre-Dame de Montréal, le 4 février 1862, folio 7; Edgar Day, «Souvenirs", 2.

51 Nous remercions Larry McNally, des Archives nationales du Canada à Ottawa, pour avoir soumis à notre attention les Canada Directory et le «Répertoire des fabricants d'appareils de chauffage du Québec jusqu'en 1867», dans Marcel Moussette, Le chauffage domestique au Canada, des origines à l'industrialisation (Québec, Presses de l'Université Laval, 1983), 173230. Ces publications se sont avérées essentielles pour la préparation d'une liste préliminaire des fonderies de fer établies par des mouleurs originaires des Forges du Saint-Maurice.

52 Selon le rôle d'évaluation de 1870 , la fonderie est la propriété de James Irwin qui l'avait longtemps dirigée à son compte. Mais en 1881, le rôle d'évaluation indique que l'établissement est la propriété d'Emmanuel Day et d'Alfred Deblois. Entre-temps, Augustin Terreau est décédé, en 1871, à l'âge de 42 ans (registre des sépultures de la paroisse Notre-Dame de Montréal, le 28 juin 1871 , folio 98 ). 
FIGURE 5

Localisation géographique des mouleurs chefs de famille originaires des Forges du Saint-Maurice dans la partie sud-ouest de Montréal. Avril 1871.

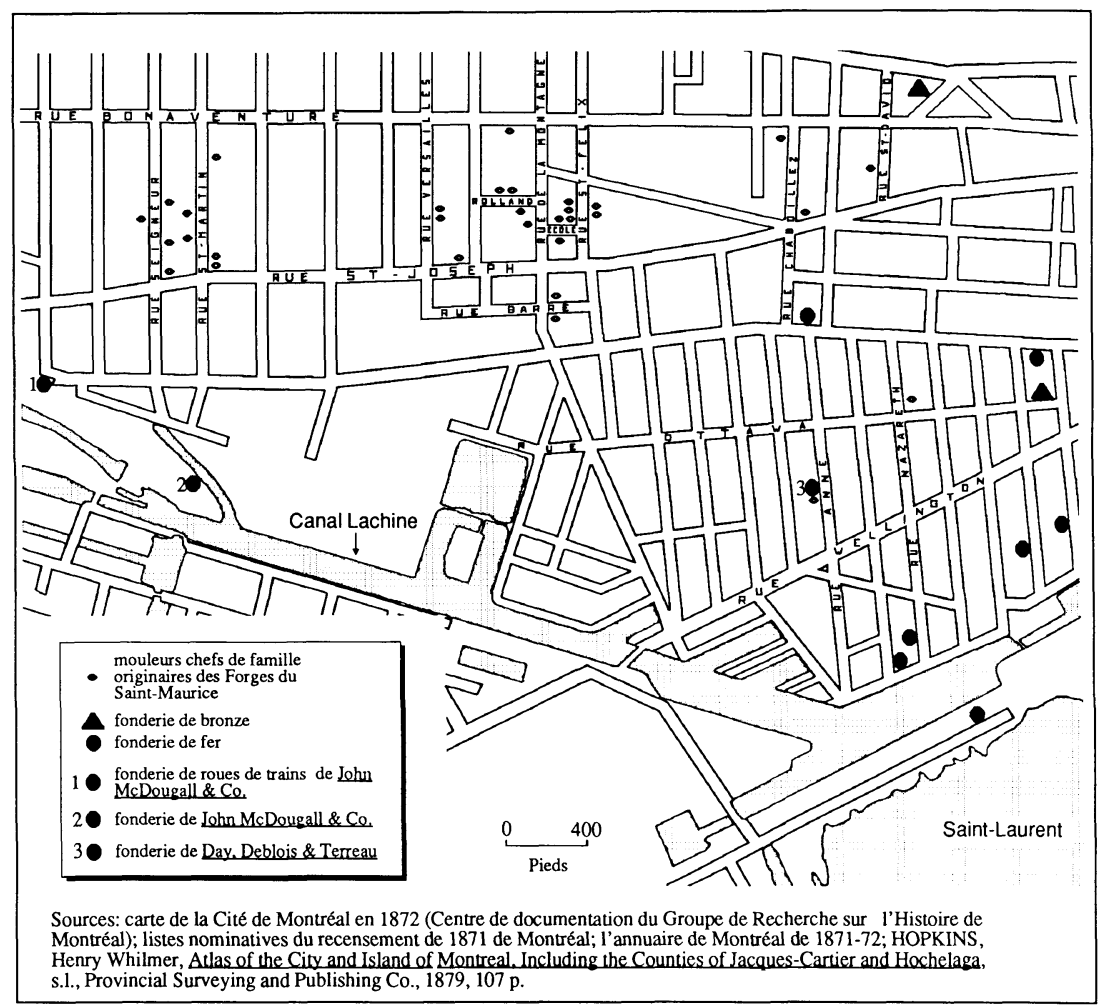

1871, cette fonderie spécialisée dans le travail du fer emploie vingtneuf travailleurs: seize d'entre eux ont moins de seize ans. Il est possible que la fonderie reproduise le caractère familial du travail qu'ont vécu les mouleurs dans les villages de Forges et les fonderies en Mauricie: les renseignements à ce sujet sont rares, mais nous savons que trois des fils d'Emmanuel Day deviennent mouleurs et travaillent dans l'établissement ${ }^{53}$.

La fonderie de roues de trains de John McDougall \& Co. et l'entreprise de Day, Terreau \& Deblois ne peuvent regrouper hypothétiquement pas beaucoup plus que la moitié des trente-neuf mouleurs ori-

\footnotetext{
53 Edgar Day, «Souvenirs», 2.
} 
TABLEAU 1

Liste préliminaire des fonderies de fer établies par des mouleurs originaires des Forges du Saint-Maurice (seuls ou en association)

Localisation

\section{Canton d'Acton \\ Ville de Beauharnois \\ Village de Hunterstown \\ Village de Joliette \\ Paroisse de Lotbinière \\ Ville de Montréal \\ Ville de Montréal}

Ville de Québec

Ville de Québec

Ville de Trois-Rivières

Ville de Trois-Rivières

Ville de Trois-Rivières

Ville de Trois-Rivières

Village de Renfrew (Ontario)
Propriétaire(s) ${ }^{1}$

Joseph Gauthier
Louis Raymond
Louis Gauthier
Pierre Imbleau
Parrot, Imbleau \& Co.
Jacques Marcotte et
Augustin Terreau
Emmanuel Day,
Alfred Deblois et
Augustin Terreau
Charles Terreau,
Guillaume Duchesneau et
Jacques Marcotte
Alphonse Terreau,
Joseph Terreau et
Louis Terreau
Louis Dupuis
Joseph Raymond
Pierre Mailloux
Samuel Sawyer et
Cléophas Viger
Luc Imbleau

\begin{tabular}{ccccc}
$\begin{array}{c}\text { Années } \\
\text { d'opération }\end{array}$ & \multicolumn{2}{c}{$\begin{array}{c}\text { Nombre } \\
\text { d'employés }\end{array}$} & \multicolumn{2}{c}{$\begin{array}{c}\text { Force } \\
\text { motrice }\end{array}$} \\
connues & 1861 & 1871 & 1861 & 1871
\end{tabular}

$1865-66$

$1861-71$

1864-65

$1846-75$

1865-66

1857

$1870-92$

29

$10 \mathrm{v}$

$1846-95$

10

$9 \mathrm{v}$

$1873-77$

$1834-61$

1852

$\begin{array}{llll}1853-71 & 3 & 2 & 2 c\end{array}$

1871-84

$1884-1947$

$\begin{array}{cccc}24 & & 10 \mathrm{v} & \\ 3 & 2 & 2 \mathrm{c} & \\ & 5 & & 6 \mathrm{v}\end{array}$

Sources: Les recensements industriels manuscrits de 1861 et 1871, les annuaires du Canada de $1851,1853,1857-58,1864-65,1865-66,1871,1890-91$ et 1947 ; les annuaires de la ville de Québec de 1855, 1857, 1861-62, 1863-64, 1872-73, 1876-77, 1878-79, 188283 ; les annuaires de Trois-Rivières de 1876, 1879, 1880 et 1884; les annuaires de Montréal de 1842 à 1895; «Répertoire des fabricants d'appareils de chauffage du Québec jusqu'en 1867», dans Marcel Moussette, Le chauffage domestique au Canada, des origines à l'industrialisation (Québec, Presses de l'Université Laval, 1983), 173-230.

\footnotetext{
1 Seuls les propriétaires dont les noms sont soulignés représentent des mouleurs originaires des Forges du Saint-Maurice.

2 Les fonderies employaient trois types de force motrice: l'eau («e»), le cheval («c») ou la vapeur $(« \mathrm{v})$. Lorsque les sources nous le permettent, nous avons inscrit l'ordre de grandeur de la force motrice utilisée: par exemple, «10v» correspond à une force de 10 chevaux-vapeur tandis que «2c" signifie que la fonderie utilise deux chevaux pour mouvoir sa machinerie.

3 À la mort de Charles Terreau, qui survient vers 1866 , l'association est dissoute. La veuve de Charles Terreau et Jacques Marcotte ouvrent alors chacun de leur côté leur propre fonderie.

4 Louis Dupuis est également co-propriétaire des forges l'Islet, avec son fils Eugène, André Robichon et Pierre Robichon. En activité de 1856 à 1862, les forges écoulent leur production de fer en gueuse à la fonderie de Dupuis à Trois-Rivières. En 1863, elles sont vendues à John McDougall, juste avant que ce dernier achète les Forges du Saint-Maurice.
} 
ginaires des Forges et présents à Montréal au recensement de 1871. Les autres sont probablement engagés, dans des proportions qui nous échappent, par les nombreuses fonderies de l'ouest de la ville produisant des poêles et autres articles en fonte. Si le maintien de la solidarité professionnelle au travail chez les mouleurs originaires des Forges du Saint-Maurice demeure conjectural, il est clair cependant qu'ils réussissent à rétablir la solidarité de voisinage dans l'espace montréalais. Ces mouleurs sont en effet installés en 1871 par «grappes» sur certaines sections de rue du quartier Saint-Antoine, soulignant d'intenses relations de voisinage entre ces familles d'ouvriers (figure 5).

Le voisinage entre familles de mouleurs situées sur les rues Seigneur et Saint-Martin au sud de la rue Bonaventure et au nord de la rue Saint-Joseph illustre bien le phénomène. Nous trouvons sur la rue Seigneur, à peu de distance les unes des autres, les familles de Pierre Imbleau, de son oncle Louis Imbleau, celles du cousin de ce dernier, Augustin Terreau, et la famille du beau-frère d'Augustin, Emmanuel Day $^{54}$. Augustin Terreau est propriétaire d'une maison à deux logements, située sur la rue Saint-Martin, dont l'un est habité par la famille de son frère Luc. Le cousin de ce dernier, Pierre Terreau, loge à côté avec sa famille. La famille du petit-cousin de Pierre Terreau, Henri Imbleau, dont le ménage inclut son père, le mouleur Pierre Imbleau, maintenant veuf, habite de biais de l'autre côté de la rue. Les deux autres familles de mouleurs domiciliées sur la rue Saint-Martin, à proximité, celles de Louis Pellerin et d'Arthur Charette, font aussi partie du réseau de parenté, mais ces liens de parenté sont plus éloignés ${ }^{55}$.

À Montréal, les relations de voisinage entre mouleurs se modifient sans cesse en raison des déménagements fréquents des familles dans l'espace urbain, ou de leurs départs et de l'arrivée d'autres familles. Cependant, en 1881, même si la localisation des familles a quelque peu changé, cette forme de sociabilité n'a pas diminué en importance ${ }^{56}$. L'analyse détaillée de la formation de ces relations de voisinage nous renvoie l'image d'un réseau communautaire fonctionnant comme un véritable système d'entraide, qui offre probablement de l'assistance aux familles migrantes pour trouver un logement. Ce réseau contribue ainsi

\footnotetext{
54 Terreau et Day sont les seuls mouleurs du réseau de parenté à Montréal propriétaires de leur demeure. Rôle d'évaluation de Montréal de 1871.

55 Montréal connaît aussi des migrations de familles originaires des Forges du Saint-Maurice dont les chefs pratiquent d'autres métiers que celui de mouleur, et de familles de mouleurs apparentés par leurs épouses aux familles de mouleurs originaires des Forges du Saint-Maurice. Mais les limites de cette recherche ne permettent pas de les inclure dans l'analyse, sauf exception.

56 La force de la sociabilité entre mouleurs originaires des Forges du Saint-Maurice s'exprime également à travers la ténacité de l'endogamie professionnelle. Malgré le fait que le marché matrimonial soit extrêmement large à Montréal, avec une population qui passe de 90323 à 140747 habitants entre 1861 et 1881 , cinq des dix-sept mariages de mouleurs originaires des Forges célébrés à Montréal continuent de s'effectuer au sein de la parenté.
} 
à l'attraction déjà très grande de Montréal sur les mouleurs originaires des Forges du Saint-Maurice et favorise leur insertion dans le nouveau milieu.

L'arrivée massive des mouleurs originaires des Forges du SaintMaurice à Montréal s'insère dans un mouvement donnant aux mouleurs canadiens-français la majorité des effectifs vers 1870 . Entre 1850 et 1870 , on assiste en fait à un bouleversement de la composition ethnique du métier. Ce dernier a longtemps été dominé par les mouleurs d'origine britannique. Cependant, constituant près de la moitié des mouleurs de la province en 1852 et 1861, les mouleurs canadiens-français affluent vers Montréal et augmentent leur importance relative dans le métier qui était de $11 \%$ en 1852 , à $35 \%$ en 1861 , et à la majorité des effectifs par la suite, même si le nombre de mouleurs passe de 38 en 1852 à 319 en 1871.

\section{B - L'action syndicale}

Lorsque ces mouleurs arrivent à Montréal, il existe alors dans cette ville, depuis 1859 , un syndicat de mouleurs dominé par des ouvriers d'origine britannique. En 1861, cette organisation joint l'Iron Molders Union of America, fondée en 1859 à Philadelphie, et devient alors la section 21 de cette association de syndicats. La coalition des différents syndicats locaux au sein d'un tel regroupement vise avant tout à faciliter les déplacements de leurs membres à la recherche de travail et à contrôler leur circulation interurbaine. Le but visé est d'éviter les engorgements des marchés de travail locaux dont les capitalistes sont les seuls à tirer profit. Ce syndicalisme marque une nouvelle phase dans l'antagonisme ouvriers/patrons, car les mouleurs syndiqués ne s'en tiennent pas à une résistance contre les prétentions des employeurs, mais ils cherchent aussi à contrôler le niveau des salaires, le processus de travail, le recrutement, l'embauche et la disponibilité de la main-d'oeuvre ${ }^{57}$.

Contrairement aux États-Unis, où les mouleurs spécialisés dans le domaine des poêles sont à l'avant-garde du mouvement de syndicalisation, à Montréal, le syndicat est fondé par les mouleurs de machinerie $^{58}$. Au cours des années suivantes, l'organisation syndicale montréalaise rallie des mouleurs d'autres fonderies et domaines de spécialisation. Des indices suggèrent qu'aux États-Unis comme au Québec, les mouleurs originaires de villages de forges jouent un rôle important dans la montée du syndicalisme ${ }^{59}$. À Montréal, les mouleurs ori-

\footnotetext{
57 Pour une analyse de la nature et de l'évolution des actions revendicatives des mouleurs montréalais, voir Peter Bischoff, «La formation des traditions...».

58 Cette information provient de souvenirs de membres fondateurs réunis à l'occasion du cinquantième anniversaire de la section 21. Alex R. Mitchell, «The Molders Union of Montreal, Quebec», IMJ, 45,7 (juillet 1909): 514-515.

59 Plusieurs membres fondateurs du premier syndicat de l'Iron Molders International Union, celui de Philadelphie, établi en 1855, figurent parmi les mouleurs ayant quitté le village de forges
} 
ginaires des Forges du Saint-Maurice se révèlent en effet sensibles à la nécessité de préserver le statut de leur métier par l'action syndicale. Il est surprenant de constater que, entre 1866 et 1870, dix d'entre eux adhèrent à un moment ou à un autre à la section 21 . Ils représentent alors une nette majorité des mouleurs canadiens-français membres du syndicat. Sept de ces dix mouleurs joignent le syndicat l'année même de leur arrivée à Montréal. Mais cinq d'entre eux se font expulser tout aussi rapidement, en 1866 et 1867, pour ne pas avoir payé leur cotisation, et seulement un, ou peut-être deux, sont membres du local jusqu'en $1881^{60}$.

La sortie de la majeure partie des mouleurs originaires des Forges du Saint-Maurice de la section 21 ne semble pas résulter d'un manque d'intérêt pour une structure permanente de défense du métier. En effet, des indices suggèrent que certains d'entre eux participent, en 1867, à l'organisation d'un corps de métier de mouleurs, membre de la Grande Association des corps de métier de Montréal. Cette Grande Association de vingt-six corps de métier, dirigée par Médéric Lanctôt, défend trois grands objectifs complémentaires: favoriser la collaboration Capital/ Travail, travailler au bien-être des ouvriers et arrêter l'immigration. Organisé en avril par Médéric Lanctôt, le corps de métier des mouleurs est dirigé majoritairement par des Canadiens français. De plus, les mouleurs de poêles semblent être particulièrement influents en son sein ${ }^{61}$. Le mouleur Luc Terreau, qui se fait expulser du syndicat en 1866, devient membre du corps de métier et est élu à l'un des six postes de l'exécutif de l'organisation: celui de trésorier. Son petit-cousin, Pierre Imbleau, qui n'a jamais été membre de la section 21 , accède à l'une des six places de délégué du corps de métier à la commission centrale de la Grande Association.

Bien que nos données soient lacunaires, on peut émettre l'hypothèse que le comportement des mouleurs originaires des Forges du SaintMaurice s'apparente, à partir de 1867, à l'attitude des mouleurs canadiens-français en général: on garde ses distances vis-à-vis de la section 21 , trop «britannique», préférant plutôt joindre le corps de métier des mouleurs membre de la Grande Association où les Canadiens français semblent être majoritaires ${ }^{62}$. Des tensions ethniques et linguistiques qui

de Hopewell (Pennsylvanie), lorsque l'établissement se spécialise dans la production de fer en gueuses, à partir de 1844. Joseph Walker, «Labor-Management Relations at Hopewell Village», Labor History, 14 (1973): 8-9 et Joseph Walker, Hopewell Village, the Dynamics of a Nineteenth Century Iron-Making Community (Philadelphie, University of Pennsylvania Press, 1966), 36.

60 Rapports de la section 21,IMJ, 1864-1881.

61 Â la procession organisée par la Grande Association le 10 juin 1867 , où plus de 10000 ouvriers des divers corps de métier manifestent publiquement leur solidarité, les mouleurs possèdent leur propre cortège qui a pour emblême un poêle. L'Union nationale, 12 juin 1867.

62 Les mouleurs canadiens-français occupent quatre des six postes de l'exécutif du corps de métier (dont celui de président) et trois des six places de délégués de l'organisation à la commission centrale de la Grande Association. L'Union nationale, 29 avril 1867. 
opposent mouleurs canadiens-français et mouleurs d'origine britannique sont à la base de cette division des rangs des mouleurs. Cette explication est corroborée par les souvenirs de membres fondateurs de la section 21, qui qualifient ces tensions de «malédiction pour la ville», car elles paralysent leur organisation ${ }^{63}$.

L'existence de la Grande Association est de courte durée et les mouleurs originaires des Forges semblent retomber rapidement dans un état d'inorganisation professionnelle. Mais est-ce réellement le cas? Dans un contexte où la voie de l'organisation est temporairement bloquée, ces mouleurs s'appuient probablement sur leur réseau communautaire qui semble constituer une importante source d'assistance pour trouver du travail. Ainsi, certains faits permettent de supposer qu'il existe un échange d'informations sur les ouvertures d'emplois entre les membres du réseau habitant Montréal et ceux situés ailleurs dans la province.

La famille du mouleur Narcisse Sawyer, par exemple, a d'abord vécu aux Forges du Saint-Maurice, en 1852, puis à Trois-Rivières en 1861. Au début des années 1870 , la famille se retrouve dans le quartier Saint-Roch de Québec et il est possible que le père travaille dans la fonderie de sa parente, «Mme Charles Terreau», située dans le même quartier $^{64}$. En 1875, la famille migre à Montréal. Elle habite pendant quatre ans au 110 rue Sainte-Anne, un immeuble situé sur le terrain de la fonderie Day \& Deblois et appartenant aux propriétaires de l'entreprise. Durant ces années, Narcisse Sawyer travaille probablement à la fonderie locale du réseau communautaire ${ }^{65}$.

Étienne Mailloux et sa famille, après avoir vécu aux Forges du Saint-Maurice en 1852, s'installent pour plusieurs années à TroisRivières, probablement dès la fermeture de l'établissement vers 1858. Derrière cette apparence de nouvelle stabilité (ils se trouvent aux recensements de 1861 et de 1871 à Trois-Rivières), ils font un bref séjour à Montréal en 1867, car, selon l'annuaire de cette ville, Étienne Mailloux habite au 24 rue Saint-Félix. Le mouleur originaire des Forges du SaintMaurice, Éloi Terreau, qui habite au 23 Saint-Félix depuis 1865, leur a probablement «donné un coup de main» pour trouver ce logement. Ils reviennent pour de brèves périodes de temps à Montréal en 18741875 et en 1878-1879 et habitent toujours dans le voisinage d'autres familles de mouleurs originaires des Forges ${ }^{66}$.

63 Alex R. Mitchell, «The Molders Union of Montreal...», 514-515.

64 Charles Terreau est décédé au milieu des années 1860 et son épouse a pris sa relève, jusqu'à sa propre retraite en 1895. Il était le cousin du père de Narcisse Sawyer. Marcel Moussette, Le chauffage domestique..., 227-228.

65 Listes nominatives de recensement du Canada de 1852 pour les Forges du Saint-Maurice et de 1861 pour Trois-Rivières; les annuaires de Québec de 1872-1873 et 1874-1875; les annuaires de Montréal de 1875-1876 à 1881-1882.

66 Listes nominatives de recensement du Canada de 1852 pour les Forges du Saint-Maurice, de 1861 et de 1871 pour Trois-Rivières; les annuaires de Montréal de 1867-1868, 1874-1875 et 1878-1879. 
Ces exemples pourraient être multipliés. Ils semblent indiquer que la communauté fonctionne en partie comme un système de placement qui oriente les mouvements des familles à travers les centres industriels. Le réseau communautaire représente ainsi, dans le contexte de division des rangs des mouleurs montréalais, une véritable force collective constituant un substitut à l'importante fonction de «balisage» du marché du travail, caractéristique du syndicalisme des mouleurs de l'époque ${ }^{67}$. Les mouleurs originaires des Forges du Saint-Maurice sont d'autant plus indépendants du syndicat que l'autonomie fonctionnelle au travail, caractéristique de leur métier, leur offre une position relativement forte vis-à-vis les employeurs ${ }^{68}$. Ces points d'appui semblent constituer une alternative viable à la voie syndicale.

Cependant, durant la décennie 1870 , plusieurs facteurs semblent entraîner une mutation graduelle de la conscience ouvrière de l'ensemble des mouleurs, et ce changement s'exprime, à partir de 1879, par un afflux très important de mouleurs dans les rangs de la section 21 . La lutte pour la réduction de la durée de la journée de travail en 1872, impliquant surtout les mouleurs des domaines de la machinerie et du bronze, constitue la première confrontation générale avec les employeurs et marque probablement une prise de conscience de l'importance de la force collective résultant de l'unité d'action des mouleurs de toutes les ethnies. La dégradation économique accentuée de la condition des mouleurs pendant la crise économique de 1874-1879 prépare un tournant dans le développement d'une conscience ouvrière chez ces travailleurs. C'est vers le renforcement d'une solidarité inter-ethnique et la canalisation de leurs forces à l'intérieur d'une organisation permanente de règlementation et de défense de leur métier, que les mouleurs s'orientent alors. Au printemps 1879, une nouvelle offensive patronale contre le métier mine sérieusement l'autonomie fonctionnelle de ces ouvriers et accélère l'évolution de leur prise de conscience. La reprise économique à l'automne 1879 marque l'adhésion massive de mouleurs non syndiqués et d'ex-mouleurs syndiqués à la section 21 . L'essor du syndicalisme s'effectue sous l'impulsion des mouleurs canadiens-français et, en seconde place, des mouleurs canadiens-irlandais ${ }^{69}$.

67 Cette conclusion s'apparente à celle formulée par Tamara Hareven sur les travailleurs de l'usine d'Amoskeag, au XXe siècle. Voir Tamara K. Hareven, «The Laborers of Manchester, New Hampshire, 1912-1922: the Role of Family and Ethnicity in Adjustment to Industrial Life», Labor History, 11,2 (Spring 1975), 262.

68 Les mouleurs possèdent longtemps une autonomie fonctionnelle considérable dans les ateliers de moulage car, ce sont eux qui planifient la façon d'effectuer leurs tâches et qui dirigent le travail de leur(s) aide(s). Cette autonomie professionnelle permet aux mouleurs non syndiqués de se montrer très indépendants du syndicat, car elle leur offre une position relativement forte au travail vis-à-vis leurs employeurs. Voir à ce sujet Peter Bischoff, «La formation des traditions...», 14-15.

69 Pour plus de détails sur ces événements, le lecteur est référé à Peter Bischoff, «La formation des traditions...», 24-37. 
Bien que nous ne connaissons pas le rôle des mouleurs originaires des Forges du Saint-Maurice dans le «mouvement des neuf heures» en 1872 (peut-être en sont-ils absents), ils sont partie prenante de cette prise de conscience ouvrière. En 1881, leurs 38 représentants à Montréal ne forment que $9 \%$ des 415 mouleurs de l'agglomération, dont $18 \%$ des effectifs canadiens-français, mais ils composent $19 \%$ des quelque 200 membres de la section et $31 \%$ de leurs membres canadiensfrançais $^{70}$.

L'élan du syndicalisme chez les mouleurs est tel que, au mois d'août 1880 , le président de la section 21 organise une nouvelle section de l'Iron Molders International Union à Trois-Rivières: la section 195 se compose de huit membres fondateurs ${ }^{71}$. La montée de la combativité des mouleurs à Montréal entretient des rapports complexes et méconnus avec les autres grands centres de la sidérurgie au Québec. Elle laisse entrevoir l'existence de liens de métier interrégionaux s'appuyant partiellement sur les informations véhiculées par les mouleurs itinérants. Les mouleurs originaires des Forges sont peut-être directement impliqués dans ce débordement de l'action revendicative. Deux des huit membres fondateurs de la section 195 sont en effet originaires des Forges du Saint-Maurice. De plus, quelques mois avant la fondation de la section trifluvienne, en avril 1880, trente mouleurs de la ville de Québec se joignent pour constituer la section 176 de l'Iron Molders International Union: deux sont les descendants des «Terreau» des Forges du SaintMaurice et l'un d'eux est élu trésorier de la section ${ }^{72}$.

\section{CONCLUSION}

L'étude des mouleurs originaires des Forges du Saint-Maurice montre que la formation de la classe ouvrière dans les centres urbains au XIXe siècle n'est pas un processus séparé, isolé du reste du territoire. Dès les décennies 1830 et 1840 , le développement de la sidérurgie de transformation $^{73}$ à Montréal, Trois-Rivières et Québec puise dans la main-d'oeuvre qualifiée formée aux Forges du Saint-Maurice, en l'occurrence, les mouleurs. Au cours des décennies suivantes, cette tendance devient plus prononcée. La sidérurgie de transformation, particulièrement les fonderies montréalaises, connaît un véritable essor, délogeant les forges de la Mauricie de la fabrication d'articles en fonte et s'accaparant la main-d'oeuvre ainsi libérée.

\footnotetext{
70 À la parade de la Saint-Jean-Baptiste, le 24 juin, près de deux cents mouleurs syndiqués marchent dans le cortège de la section. Lettre ouverte du correspondant au journal de la section 21 à l'éditeur, $I M J$, juin 1881 .

71 L'existence de la section 195 est brève: d'août 1880 à mars 1881 . Rapports de la section $195, I M J$, août 1880 à mars 1881 .

72 La section 176 opère d'avril 1880 à mars 1888 . Rapports de la section $176, I M J$, avril 1880 à mars 1888 .

73 Nous faisons référence ici à l'ensemble des activités reliées à la production d'articles à partir d'une fonte de seconde fusion.
} 
Cependant, les mouleurs originaires des Forges du Saint-Maurice ne constituent pas des victimes passives de ces bouleversements socioéconomiques. Les migrations qu'ils effectuent ne marquent pas non plus la désintégration de la sociabilité développée aux Forges. Ces déplacements s'inscrivent au contraire dans un projet axé sur la préservation de la pratique de leur métier, où ils tentent de façonner le nouveau contexte de travail à leurs besoins. Ils vont s'appuyer sur leur héritage communautaire pour faire face aux changements socio-économiques qu'entraîne l'industrialisation. Cet héritage se compose non seulement de la pratique d'un métier mais aussi de liens sociaux développés aux Forges du Saint-Maurice.

Il faut bien comprendre que les Forges du Saint-Maurice n'ont jamais été ce milieu fermé décrit par Pentland ${ }^{74}$. Dès le début du XIXe siècle, et peut-être même avant, les mouleurs originaires de ces Forges ont travaillé dans d'autres établissements sidérurgiques de la Mauricie ou à l'extérieur de la région. La solidarité communautaire a été un facteur central dans leur adaptation aux nouveaux milieux qu'ils ont pénétrés. Au cours des décennies 1850 et 1860 , les liens communautaires demeurent toujours au coeur de la réponse des mouleurs au développement des possibilités de travail dans d'autres localités de la province. Mais devant la fermeture des ateliers de moulage dans les villages de forges de la Mauricie, ces relations acquièrent une importance encore plus grande. Elles vont alors constituer le fondement d'un vaste réseau communautaire, formant un système d'échanges d'information et d'entraide, qui couvre le coeur de la nouvelle sidérurgie québécoise de transformation, Montréal, et d'autres centres secondaires.

L'expérience des mouleurs originaires des Forges du Saint-Maurice s'inscrit dans un contexte plus vaste, celui de la spécialisation, puis de la disparition des villages de forges en Amérique du Nord. Dès la fin des années 1830 , les haut-fourneaux de la Pennsylvanie affrontent la concurrence des fonderies urbaines et doivent les uns après les autres se retirer de la fabrication d'articles de fonte pour se consacrer exclusivement à la production de fer en gueuse ${ }^{75}$. Au Québec, cette situation apparaît au cours de la décennie suivante. Aux États-Unis comme au Québec, les mouleurs ainsi libérés affluent vers les centres urbains. Du côté américain, ces mouleurs semblent s'impliquer très tôt, et avec succès, dans le mouvement de syndicalisation. Au Québec, les mouleurs montrent des dispositions similaires qui sont cependant tempérées longtemps par les tensions ethniques et linguistiques locales.

\footnotetext{
74 H. C. Pentland, Labour and Capital in Canada..., section «The St. Maurice Workmen», 42-46.

Joseph Walker, «Labor-Management Relations...», 3-4.
} 